\title{
Efeito do Tratamento de Sementes de Milho com Inseticidas Sobre o Rendimento de Grãos
}

\author{
Ivan Cruz
}

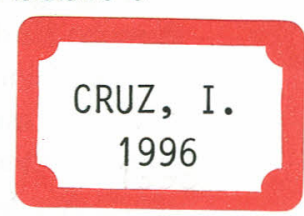

${ }^{1}$ EMBRAPA/CNPMS, Caixa postal 151, 35701-970, Sete Lagoas, MG.

An. Soc. Entomol. Brasil 25(2): 181-189 (1996)

Effect of Seed Treatment with Insecticides on Maize Yield

\begin{abstract}
One of the factors decreasing maize yield in Brazil is related to the soil insect complex and the lesser corn stalkborer, Elasmopalpus lignosellus (Zeller). This study was conducted to evaluate carbofuran, carbosulfan and thiodicarb, alone or in mixture with micronutrients (boron, zinc and molybdenum), as seed treatment to control the initial pests of maize, using the hybrid BR 201. Evaluations were based on plant emergence, insect damage and yield parameters. The dosage of each product was $700 \mathrm{~g}$ of active ingredient / 100 $\mathrm{kg}$ of seed. Except for carbaryl, the use of insecticides as a seed treatment increased the number of plants per unit area up to $45 \%$, compared to the check. The average maize yield from treated plots varied from 818 to $1414 \mathrm{~kg} /$ ha, greater than the yield obtained from the check plots.
\end{abstract}

KEY WORDS: Insecta, chemical control, soil insects, Elasmopalpus lignosellus, Spodoptera frugiperda.

RESUMO - Um dos fatores que provoca o baixo rendimento de milho no Brasil está relacionado às pragas subterrâneas e a Elasmopalpus lignosellus (Zeller). Este estudo teve como objetivo avaliar carbofuran, carbosulfan e thiodicarb, sozinhos ou em mistura com micronutrientes. Foram utilizados tratamentos e repetições em blocos ao acaso, utilizando o milho BR 201. As avaliações basearam-se na emergência das plantas, no ataque das pragas e nos parâmetros de produção. A dosagem de cada produto foi de $700 \mathrm{~g}$ do ingrediente ativo/100 $\mathrm{kg}$ de sementes. Exceto para o carbaryl, a utilização de inseticida misturados à sementes, propiciou um aumento no número de plāntas de até a $45 \%$ em relação a testemunha sem tratamento. Os rendimentos médios obtidos de parcelas tratadas variaram de 818 a $1.414 \mathrm{~kg} / \mathrm{ha}$, acima do rendimento obtido das parcelas sem tratamento.

PALAVRAS-CHAVE: Insecta, controle químico, pragas subterrâneas, Elasmopalpus lignosellus, Spodoptera frugiperda. 
Logo após o plantio as sementes ou raízes de milho são danificadas por insetos que vivem no solo, diminuindo diretamente a produtividade por ocasionar morte ou, indiretamente, por tornar a planta menos competitiva. Da emergência da plântula até os primeiros 30 dias do plantio, a lagarta-elasmo, Elasmopalpus lignosellus (Zeller) e a lagarta-do-cartucho, Spodoptera frugiperda (Smith) reduzem a produtividade por causar a morte da planta. Quantitativamente os dados sobre perdas causadas pelas pragas subterrâneas são escassos. Cruz et al. (1983), mostraram que o número de plantas emergidas de sementes sem inseticidas, foi em média $10 \%$ inferior àquele obtido das sementes tratadas com os inseticidas mais eficientes. No local estudado predominam cupins, larva-arame e bicho bolo (Waquil et al. 1992). Para a lagarta-elasmo, há um aumento de 2,8\% no rendimento de grãos para cada $1 \%$ de controle (All et al. 1979). Cruz et al. (1983) encontraram diferenças acima de $1500 \mathrm{Kg} / \mathrm{ha}$, entre parcelas de milho tratadas com inseticidas por ocasião do plantio, e não tratadas. Para a lagartado-cartucho já foram assinalados perdas de até 34\% (Carvalho 1970, Cruz \& Turpin 1982, 1983).

As opções para o controle dessas pragas iniciais do milho não são muitas. Controle curativo não tem sido eficiente. Especi- ficamente para as pragas subterrâneas, o controle químico é preventivo porque sistemas de amostragens não são eficientes. Diante dessas considerações aliadas à facilidade do tratamento de sementes e relativamente custo baixo, tem-se investido em pesquisas sobre este assunto.

O objetivo desse trabalho foi avaliar os ingredientes ativos carbofuran, carbosulfan e thiodicarb, sozinhos, ou em mistura com micronutrientes (boro, zinco e molibdênio), todos em formulação para tratamento de sementes.

\section{Material e Métodos}

Os experimentos foram realizados em Sete Lagoas e Janaúba, MG em área experi- mental da EMBRAPA, no Centro Nacional de Pesquisa de Milho e Sorgo. Os micronutrientes utilizados foram zinco, boro, e molibdênio, em concentrações recomendadas pelo fabricante. Para cada inseticida, foi utilizado um $\mathrm{kg}$ de sementes as quais foram colocadas em sacos de plástico transparente de cinco $\mathrm{kg}$ de capacidade e a seguir foi adicionado o produto. Foi realizada homogeneização por cerca de cinco minutos, até que todas as sementes estivessem uniformemente cobertas. O plantio foi efetuado no dia seguinte ao tratamento, nos experimentos de Sete Lagoas e dois dias após, no experimento de Janaúba. Foi utilizado o delineamento experimental de blocos ao acaso com o número de repetições variável com cada experimento, utilizando o híbrido BR 201, em espaçamento de um metro entre fileiras com cinco sementes por metro em plantio manual. No ano agrícola de 1991/92 o experimento foi conduzido em Sete Lagoas com sete tratamentos e cinco repetições, sendo cada parcela composta de quatro fileiras de $7 \mathrm{~m}$, sendo o plantio realizado em 17/10/91. O segundo experimento realizado em Sete Lagoas, o plantio foi efetuado em janeiro de 1993, com oito tratamentos e cinco repetições, incluindo uma formulação menos concentrada de thiodicarb (Futur), além de carbaryl (Sevin). A parcela experimental foi de quatro fileiras de $5 \mathrm{~m}$. Em Janaúba o experimento foi realizado no dia 14 de maio de 1992, com nove tratamentos e seis repetições. Cada parcela foi composta de quatro fileiras de $5 \mathrm{~m}$. As avaliações basearam-se no número de plantas emergidas, no ataque da lagarta-elasmo e lagarta-do-cartucho, no número de plantas e espigas na colheita e no rendimento de grão. Para se avaliar o ataque da lagarta-elasmo foram realizadas contagens de dois em dois dias, do número de plantas mortas e também do número de plantas sobreviventes. Para a lagarta-do-cartucho, avaliou-se a percentagem de plantas atacadas. 


\section{Resultados e Discussão}

Aos 12 dias após o plantio (DAP), a percentagem de emergência de plantas no primeiro experimento de Sete Lagoas variou de $75,3 \%$ (testemunha) a $89,6 \%$ (parcelas cujas sementes foram tratadas com Semevin + Micronutrientes) (Tabela 1). A média geral de emergência foi de $83,4 \%$. O plantio nesta região, quando realizado em outubro, número de plantas entre as duas avaliações, variou de $1,8 \%$ (parcelas tratadas com Semevin + micronutrientes) a $17,4 \%$ (parcelas testemunhas). A queda no número de plantas foi de $5 \%$ para as parcelas tratadas com Semevin, 7,1\% para Furadan e 5,3\% para o Furazin. Aos 32 DAP, a queda percentual foi: testemunha $20,4 \%$, Semevin + micronutrientes, 3,02\%; Semevin + micronutrientes + peridian, 4,7; Semevin, 6,3\%; Furazin, 7,6

Tabela 1. Número de plantas de milho obtido de parcelas sujeitas a diferentes tratamentos com inseticidas misturadas às sementes, Sete Lagoas, 1992.

\begin{tabular}{|c|c|c|c|c|c|c|c|c|}
\hline \multirow[t]{2}{*}{$\begin{array}{l}\text { Produto comercial } \\
\text { (p.c.) }\end{array}$} & \multirow[t]{2}{*}{ Princípio ativo } & \multirow{2}{*}{$\begin{array}{l}\text { Litros p.c./ } \\
100 \mathrm{~kg} \text { de } \\
\text { sementes }\end{array}$} & \multicolumn{3}{|c|}{$\begin{array}{l}\text { Número de plantas/parcela }{ }^{2,3} \\
\text { Dias após o plantio }\end{array}$} & \multicolumn{3}{|c|}{$\begin{array}{c}\text { Valor Relativo } \\
\text { Dias após o plantio } \\
\end{array}$} \\
\hline & & & 12 & 22 & 32 & 12 & 22 & 32 \\
\hline
\end{tabular}

Testemunha

$105,5 \pm 4,76 \mathrm{~d} \quad 87,2 \pm 8,50 \mathrm{~d} \quad 84,0 \pm 8,96 \mathrm{~d}$

100100100

Semevin 350+Micro Thiodicarb $+\mathrm{Zn}+$

$\mathrm{B}+\mathrm{Mo} \quad 2,001 \quad 125,5 \pm 1,26 \mathrm{a} \quad 123,2 \pm 1,76 \mathrm{a} \quad 121,7 \pm 1,87 \mathrm{a} \quad 117141145$

Semevin $350 \mathrm{SC} \quad$ Thiodicarb $+\mathrm{Zn}+$

\begin{tabular}{|c|c|c|c|c|c|c|}
\hline & $\mathrm{B}+\mathrm{Mo}$ & 2,001 & $121,2 \pm 1,08 \mathrm{ab}$ & $117,0 \pm 0,84 \mathrm{ab}$ & $115,5 \pm 0,96 a b$ & 115134137 \\
\hline Semevin $350 \mathrm{SC}$ & Thiodicarb & 2,001 & $113,5 \pm 2,74 \mathrm{bcd}$ & $107,8 \pm 2,74 b c$ & $106,3 \pm 2,60 b c$ & 107124126 \\
\hline Furazin $310 \mathrm{TS}$ & Carbofuran $+\mathrm{Zn}$ & 2,251 & $118,5 \pm 1,61 \mathrm{abc}$ & $112,2 \pm 2,24 b c$ & $109,5 \pm 2,20 \mathrm{bc}$ & 112129130 \\
\hline Furadan $350 \mathrm{SC}$ & Carbofuran & 2,001 & $110,5 \pm 4,46 \mathrm{~cd}$ & $102,7 \pm 2,85 c$ & $99,7 \pm 3,15 \mathrm{c}$ & 105110119 \\
\hline $\begin{array}{l}\text { Semevin } 350 \mathrm{SC} \\
+ \text { Peridian }\end{array}$ & $\begin{array}{l}\text { Thiodicarb + } \\
\text { peridian }\end{array}$ & 2,001 & $123,2 \pm 2,48 a$ & $117,8 \pm 2,56 \mathrm{ab}$ & $115,3 \pm 2,98 \mathrm{ab}$ & 117135137 \\
\hline
\end{tabular}

CV $(\%)$

5,6

7,6

8,2

\begin{abstract}
${ }^{1}$ Peridian é um adjuvante.
${ }^{2}$ Máximo de 140 plantas.

$\approx$ Médias seguidas pela mesma letra na coluna, não diferem significativamente entre si, nível de $5 \%$ segundo o teste de Duncan.
\end{abstract}

normalmente está sujeito ao ataque da lagartaelasmo. Já na primeira avaliação da emergência das plantas foram encontradas plantas atacadas. Aos 22 DAP, a queda no
$\%$; Furadan, 9,8\%; e Semevin + peridian, $6,4 \%$. Ainda pela Tabela 1 , fazendo a comparação entre os tratamentos, observamse variações percentuais a maior, de 5 a $19 \%$, 
nas parcelas tratadas em relação a testemunha. $\mathrm{O}$ maior percentual foi obtido das parcelas tratadas com o Semevin + micronutrientes. Com o passar do tempo e com a ocorrência da lagarta-elasmo as diferenças em relação à testemunha tornaram-se maiores, variando de 19 a $45 \%$.

$\mathrm{Na}$ primeira avaliação do ataque da lagarta-do-cartucho, realizada 10 dias após a emergência, $63,4 \%$ das plantas nas parcelas testemunhas apresentavam-se com algum dano do inseto; percentagem de dano semelhante foi observada nas parcelas em que as sementes foram tratadas com Furadan e Furazin. As menores percentagens foram encontradas nas parcelas tratadas com Semevin e com Semevin + micronutrientes. $\mathrm{Na}$ segunda avaliação realizada 10 dias após a primeira já não se observou diferenças entre as parcelas; provavelmente a ação dos ocorreu para o número de espigas e rendimento de grãos (Tabela 3 ).

O segundo experimento, realizado em Sete Lagoas, foi conduzido numa época que normalmente se tem alta infestação da lagartaelasmo. A percentagem média de emergência foi acima de $83 \%$ para todas as parcelas, exceto para aquela em que as sementes foram tratadas com Sevin 480. Este produto apresentou um efeito tóxico, diminuindo significativamente a germinação. Também não manteve a população de plantas que germinaram, em relação ao número de plantas estabelecido (após cessar o ataque da lagartaelasmo). Não houve diferença significativa entre os demais produtos (Tabela 4). Esta mesma situação ocorreu para os parâmetros, número de plantas mortas e número de plantas obtido na colheita. $\mathrm{O}$ menor rendimento foi obtido das parcelas cujas sementes foram

Tabela 2. Percentagem de infestação por lagartas de Spodoptera frugiperda em parcelas de milho sujeitas a diferentes tratamentos com inseticidas misturados às sementes, Sete Lagoas, 1992.

\begin{tabular}{llcl}
\hline Produto Comercial & \multicolumn{2}{c}{ Dias Após a Germinação } & Média $^{1}$ \\
\cline { 2 - 3 } & \multicolumn{1}{c}{10} & 20 & \\
\hline Testemunha & $63,4 \pm 5,71 \mathrm{a}$ & $36,2 \pm 4,35$ & $49,8 \pm 5,35$ \\
Semevin 350 SC + Micro & $16,8 \pm 3,73 \mathrm{c}$ & $27,8 \pm 3,61$ & $22,3 \pm 2,98$ \\
Semevin 350 SC + Micro + Peridian & $26,9 \pm 2,03 \mathrm{bc}$ & $31,3 \pm 2,34$ & $29,1 \pm 1,62$ \\
Semevin 350 SC & $36,3 \pm 3,68 \mathrm{~b}$ & $39,9 \pm 4,01$ & $38,1 \pm 2,65$ \\
Furazin 310 TS & $55,1 \pm 5,43 \mathrm{a}$ & $42,9 \pm 2,95$ & $49,0 \pm 3,48$ \\
Furadan 350 SC & $66,7 \pm 4,93 \mathrm{a}$ & $46,4 \pm 3,11$ & $56,5 \pm 4,72$ \\
Semevin 350 + Peridian & $38,8 \pm 4,23 \mathrm{~b}$ & $35,9 \pm 3,37$ & $37,3 \pm 2,62$ \\
& & & \\
\hline CV $(\%)$ & 26,0 & 24,9 & \\
\hline
\end{tabular}

${ }^{1}$ Médias seguidas pela mesma letra na coluna não diferem significativamente entre si, ao nível de $5 \%$ segundo o teste de Duncan.

produtos já não era eficiente nesta ocasião (Tabela 2).

O número de plantas obtido na colheita variou de 28.678 a 40.786 por hectare, sendo o menor número proveniente da testemunha, que diferiu significativamente de todos os outros tratamentos. Situação semelhante tratadas com Sevin 480 seguido do rendimento obtido das parcelas testemunhas ( $2.367 \mathrm{~kg} / \mathrm{ha})$. Os rendimentos das demais parcelas foram significativamente se- melhantes, com média de $3.185 \mathrm{~kg} / \mathrm{ha}$ (Tabela 4).

Em Janaúba, MG, aos 13 DAP, a emergência das plantas nas parcelas sem 
Tabela 3. Número de plantas, número de espigas e rendimentos de grãos obtidos de parcelas sujeitas a diferentes tratamentos com inseticidas misturados às sementes de milho, Sete Lagoas, 1992.

\begin{tabular}{|c|c|c|c|}
\hline Tratamento & $\begin{array}{l}\text { Plantas/ha }{ }^{1} \\
\text { (x 1000) }\end{array}$ & $\begin{array}{l}\text { Espigas/ha }^{1} \\
\quad(\mathrm{x} 1000)\end{array}$ & $\begin{array}{c}\text { Rendimentos }{ }^{1} / \mathrm{kg} / \mathrm{ha} \\
(\mathrm{x} 1000)\end{array}$ \\
\hline Testemunha & $28,7 \pm 3,05 \mathrm{~d}$ & $39,6 \pm 1,82 \mathrm{c}$ & $4,7 \pm 0,34 \mathrm{c}$ \\
\hline Semevin + Micro & $40,8 \pm 1,00 \mathrm{a}$ & $50,1 \pm 1,67 \mathrm{a}$ & $6,2 \pm 0,20 \mathrm{a}$ \\
\hline Semevin + Micro + Peridian & $38,7 \pm 0,77 a b$ & $48,3 \pm 1,12 \mathrm{ab}$ & $5,9 \pm 0,09 \mathrm{ab}$ \\
\hline Semevin & $36,1 \pm 0,91 b c$ & $48,2 \pm 0,93 a b$ & $5,7 \pm 0,15 \mathrm{ab}$ \\
\hline Furazin & $36,8 \pm 0,74 b c$ & $47,5 \pm 1,50 \mathrm{ab}$ & $5,6 \pm 0,18 \mathrm{ab}$ \\
\hline Furadan & $34,2 \pm 1,19 c$ & $45,5 \pm 1,23 b$ & $5,3 \pm 0,23 b$ \\
\hline Semevin + Peridian & $37,7 \pm 0,71 \mathrm{abc}$ & $47,1 \pm 1,46 a b$ & $5,5 \pm 0,21 b$ \\
\hline$\overline{C V} .(\%)$ & 8,2 & 7,4 & 9,6 \\
\hline
\end{tabular}

${ }^{1}$ Médias seguidas pela mesma letra na coluna não diferem significativamente entre si, ao nível de $5 \%$, segundo o teste de Duncan.

tratamento das sementes foi de $52,2 \%$. As maiores emergências foram obtidas quando as sementes foram tratadas com Semevin + micronutrientes $(73,3 \%)$, Furazin
(71,7\%), Ralzer a 2,0 $1(65,2 \%)$ e Marshall $(67,3 \%)$. Apesar da baixa percentagem de emergência, quando se compara entre os tratamentos observam diferenças desde 12

Tabela 4. Efeito de diferentes inseticidas misturados a sementes de milho, sobre a lagarta-elasmo, Elasmopalpus lignosellus, no número de plantas e nos rendimentos, Sete Lagoas, 1993.

\begin{tabular}{|c|c|c|c|c|c|c|}
\hline \multirow[t]{2}{*}{$\begin{array}{l}\text { Produto com. } \\
\text { (p.c.) }\end{array}$} & \multirow[t]{2}{*}{ Princípio ativo } & \multirow[t]{2}{*}{$\begin{array}{c}\text { Litros p.c./ } \\
100 \mathrm{~kg} \\
\text { sementes }\end{array}$} & \multicolumn{2}{|c|}{$\begin{array}{l}\text { Número de plantas } \\
\text { por parcela } \\
\text { Dias após o plantio }\end{array}$} & \multirow[t]{2}{*}{$\begin{array}{l}\text { Plantas } \\
\text { mortas }^{2,3}\end{array}$} & \multirow[t]{2}{*}{$\begin{array}{l}\text { Redimento } \\
\qquad \mathrm{kg} / \mathrm{ha}^{2} \\
(\mathrm{x} 1000)\end{array}$} \\
\hline & & & 13 & 28 & & \\
\hline Testemunha & & & $83,0 \pm 1,48 b c$ & $55,8 \pm 4,15 b$ & $25,2 \pm 4,07 \mathrm{a}$ & $2,4 \pm 0,20 \mathrm{~b}$ \\
\hline Semevin $350 \mathrm{SC}$ & Thiodicarb & 2,00 & $83,0 \pm 1,76 \mathrm{c}$ & $78,0 \pm 2,98 \mathrm{a}$ & $5,4 \pm 1,89 b$ & $3,2 \pm 0,19 a$ \\
\hline \multirow[t]{2}{*}{ Futur $300 \mathrm{SC}$} & Thiodicarb $+\mathrm{Zn}+$ & & & & & \\
\hline & $\mathrm{B}+\mathrm{Mo}$ & 2,00 & $86,4 \pm 1,33 a b c$ & $81,0 \pm 2,07 \mathrm{a}$ & $5,2 \pm 1,62 b$ & $3,3 \pm 0,25 a$ \\
\hline \multirow[t]{2}{*}{ Futur $300 \mathrm{SC}$} & Thiodicarb $+\mathrm{Zn}+$ & & & & & \\
\hline & $\mathrm{B}+\mathrm{Mo}$ & 2,33 & $90,0 \pm 1,14 \mathrm{a}$ & $80,6 \pm 4,13 a$ & $9,8 \pm 3,92 b$ & $3,3 \pm 0,35 a$ \\
\hline Furazin $310 \mathrm{TS}$ & Carbofuran $+\mathrm{Zn}$ & 2,25 & $88,4 \pm 1,29 a b c$ & $78,2 \pm 1,11 \mathrm{a}$ & $8,8 \pm 0,49 b$ & $3,1 \pm 0,10 a$ \\
\hline Sevin $480 \mathrm{SC}$ & Carbaryl & 2,00 & $76,6 \pm 3,19 \mathrm{~d}$ & $49,4 \pm 3,07 b$ & $26,4 \pm 4,48 a$ & $1,9 \pm 0,19 c$ \\
\hline Ralzer 350 SC & Carbofuran & 2,00 & $84,8 \pm 1,85 \mathrm{abc}$ & $77,6 \pm 3,23 a$ & $8,6 \pm 3,37 \mathrm{~b}$ & $2,9 \pm 0,26 a$ \\
\hline Furadan $350 \mathrm{SC}$ & Carbofuran & 2,00 & $89,0 \pm 1,38 \mathrm{ab}$ & $78,6 \pm 1,99 a$ & $10,2 \pm 1,08 b$ & $3,3 \pm 0,17 a$ \\
\hline CV. $(\%)$ & & & 4,9 & 8,3 & 25,4 & 12,2 \\
\hline
\end{tabular}

${ }^{1}$ Máximo de 100 plantas.

${ }^{2}$ Médias seguidas pela mesma letra na coluna, não diferem significativamente ao nível de $5 \%$, segundo o teste de Duncan.

${ }^{3}$ Análise feita com a transformação raiz quadrada de $(x+0,5)$. 
até $40 \%$ favoráveis ao tratamento das sementes. Os maiores valores foram obtidos de parcelas que receberam tratamentos com inseticidas misturados a micronutrientes: Semevin + micronutrientes $(40,0 \%)$ e Furazin (37\%) (Tabela 5). Para a lagarta-docartucho, numa avaliação realizada 28 DAP., os produtos, Semevin a 2,0 litros e o Ralzer a 3,0 litros / $100 \mathrm{~kg}$ de sementes dosagens), Furadan, Furazin e Marshall (Tabela 6).

O uso de inseticidas sistêmicos aplicados como tratamento de sementes tem sido salientado como o meio mais prático e econômico de reduzir os danos de pragas iniciais da cultura da soja (Petty 1967), incluindo Diabrotica sp., Agrotis ipsilon (Hufnagel), Elasmopalpus lignosellus (Zeller)

Tabela 5. Número de plantas de milho e percentagem de ataque da lagarta do cartucho, Spodoptera frugiperda, obtidos de parcelas sujeitas a diferentes tratamentos com inseticidas misturados às sementes, Janaúba, 1992.

\begin{tabular}{|c|c|c|c|c|c|c|c|}
\hline \multirow[t]{3}{*}{$\begin{array}{l}\text { Produto comerc. } \\
\text { (p.c.) }\end{array}$} & \multirow[t]{3}{*}{$\begin{array}{l}\text { Princípio } \\
\text { ativo }\end{array}$} & \multirow{3}{*}{$\begin{array}{c}\text { Litros p.c./ } \\
100 \mathrm{~kg} \mathrm{de} \\
\text { sementes }\end{array}$} & \multirow{2}{*}{\multicolumn{2}{|c|}{$\begin{array}{l}\begin{array}{l}\text { Número real de } \\
\text { plantas/parcelas }\end{array} \\
\text { Dias após plantio }\end{array}$}} & \multirow{2}{*}{\multicolumn{2}{|c|}{$\begin{array}{l}\text { Número relativo de } \\
\text { plantas / parcela } \\
\text { Dias após plantio }\end{array}$}} & \multirow{3}{*}{$\begin{array}{l}\text { Plantas com danos } \\
\frac{(\%)^{2}}{\text { Dias após plantio }} \\
28\end{array}$} \\
\hline & & & & & & & \\
\hline & & & 13 & 28 & 13 & 28 & \\
\hline \multicolumn{3}{|l|}{ Testemunha } & $52,2 \pm 10,00 \mathrm{~b}$ & $50,3 \pm 7,51 \mathrm{~b}$ & 100 & 100 & $77,0 \pm 6,24 a$ \\
\hline \multirow{2}{*}{$\begin{array}{l}\text { Semevin } 350 \text { CS } \\
+ \text { Micro }\end{array}$} & Thiodicarb & 2,00 & $73,3 \pm 1,93 \mathrm{a}$ & \multirow[t]{2}{*}{$70,8 \pm 1,02 \mathrm{a}$} & 140 & 141 & $25,3 \pm 9,11 \mathrm{~d}$ \\
\hline & \multicolumn{2}{|l|}{$+\mathrm{Zn}+\mathrm{B}+\mathrm{Mo}$} & & & & & \\
\hline Semevin $350 \mathrm{SC}$ & Thiodicarb & 2,00 & $68,0 \pm 3,96 a$ & $66,0 \pm 3,18 \mathrm{a}$ & 130 & 131 & $33,5 \pm 5,88 \mathrm{~cd}$ \\
\hline Ralzer 350 SC & Carbofuran & 2,00 & $65,2 \pm 2,52 \mathrm{ab}$ & $63,2 \pm 1,98 \mathrm{ab}$ & 125 & 123 & $60,1 \pm 4,49 a b$ \\
\hline Ralzer $350 \mathrm{SC}$ & Carbofuran & 2,50 & $58,7 \pm 4,78 \mathrm{ab}$ & $56,5 \pm 3,74 \mathrm{ab}$ & 112 & 112 & $60,4 \pm 3,84 \mathrm{ab}$ \\
\hline Ralzer 350 SC & Carbofuran & 3,00 & $58,0 \pm 3,70 \mathrm{ab}$ & $57,3 \pm 2,84 \mathrm{ab}$ & 113 & 114 & $31,1 \pm 3,01 d$ \\
\hline Marshal $250 \mathrm{TS}$ & Carbosulfan & 2,80 & $67,3 \pm 4,33 a$ & $66,8 \pm 3,48 \mathrm{a}$ & 129 & 133 & $56,9 \pm 8,43 b$ \\
\hline \multirow[t]{2}{*}{ Furazin $310 \mathrm{TS}$} & Carbofuran & 2,25 & $71,7 \pm 3,84 a$ & $70,7 \pm 2,90 \mathrm{a}$ & 137 & 140 & $57,9 \pm 4,55 b$ \\
\hline & $+\mathrm{Zn}$ & & & & & & \\
\hline Furadan $350 \mathrm{SC}$ & Carbofuran & 2,00 & $64,8 \pm 3,22 \mathrm{ab}$ & $63,0 \pm 3,21 \mathrm{ab}$ & 124 & 125 & $49,5 \pm 4,86 b c$ \\
\hline \multicolumn{3}{|l|}{$\mathrm{CV}(\%)$} & 17,3 & 17,3 & & & 28,8 \\
\hline
\end{tabular}

${ }^{1}$ Máximo de 100 plantas.

${ }^{2}$ Médias seguidas pela mesma letra na coluna não diferem significativamente entre si, ao nível de $5 \%$ segundo o teste de Duncan.

foram os mais eficientes no seu controle (Tabela 5).

Para os parâmetros obtidos na colheita, $o$ melhor resultado foi obtido de parcelas cujas sementes foram tratadas com Semevin + micronutrientes. $\mathrm{O}$ rendimento daquelas parcelas eqüivaleu a $6.738 \mathrm{~kg} / \mathrm{ha}$. Este rendimento foi estatisticamente semelhante ao obtido das parcelas cujas sementes foram tratadas com Semevin, Ralzer (duas menores e Spodoptera frugiperda (Smith) pragas essas também da cultura do milho. Segundo Robertson (1991) os insetos pragas que vivem no solo, podem ser classificados em três categorias: insetos subterrâneos que atacam a semente em processo de germinação e as raízes em formação; insetos de atividade na superfície do solo que atacam as plântulas e insetos que danificam as raízes de plantas já estabilizadas. Os insetos das duas primeiras 
Tabela 6. Número de plantas, número de espigas e rendimentos de grãos obtidos de parcelas sujeitas a diferentes tratamentos com inseticidas misturados às sementes de milho, Janaúba, 1992.

\begin{tabular}{|c|c|c|c|c|}
\hline Produto comercial (p.c.) & $\begin{array}{l}\text { Litros p.c./ } \\
100 \mathrm{~kg} \text { de } \\
\text { sementes }\end{array}$ & $\begin{array}{l}\text { Plantas/ha }{ }^{1} \\
\text { (x 1000) }\end{array}$ & $\begin{array}{c}\text { Espigas/ha' }{ }^{1} \\
\quad(\text { x 1000) }\end{array}$ & $\begin{array}{c}\text { Rendimento }(\mathrm{kg} / \mathrm{ha})^{1} \\
(\mathrm{x} 1000)\end{array}$ \\
\hline
\end{tabular}

Testemunha

Semevin $350 \mathrm{SC}+$ Micro

Semevin $350 \mathrm{SC}$

Ralzer 350 SC

Ralzer 350 SC

Ralzer 350 SC

Marshall 250 TS

Furazin 310 TS

Furadan 350 SC

2,00
2,00
2,00
2,50
3,00
2,80
2,25
2,00

$$
24,9 \pm 4,93 \mathrm{c}
$$

$35,0 \pm 1,12 \mathrm{a}$

$32,9 \pm 2,11 \mathrm{ab}$

$31,1 \pm 1,31 \mathrm{abc}$

$27,5 \pm 2,44 \mathrm{bc}$

$28,1 \pm 1,94 \mathrm{abc}$

$33,2 \pm 5,50 \mathrm{~b}$

$4,6 \pm 0,77 \mathrm{c}$

$46,7 \pm 1,94 \mathrm{a}$

$32,9 \pm 2,11 \mathrm{~b}$

$6,7 \pm 0,40 \mathrm{a}$

$31,1 \pm 1,31 \mathrm{~b}$

$6,4 \pm 0,43 \mathrm{ab}$

$27,5 \pm 2,44 \mathrm{~b}$

$6,2 \pm 0,33 \mathrm{ab}$

$28,1 \pm 1,94 \mathrm{~b}$

$5,3 \pm 0,74 \mathrm{abc}$

$5,2 \pm 0,46 \mathrm{bc}$

$6,1 \pm 0,47 \mathrm{abc}$

$32,8 \pm 1,89 \mathrm{ab}$

$32,8 \pm 1,89 \mathrm{~b}$

$34,0 \pm 1,36 \mathrm{~b}$

$6,4 \pm 0,34 \mathrm{ab}$

$34,0 \pm 1,53 \mathrm{ab}$

$30,4 \pm 1,47 \mathrm{~b}$

$6,1 \pm 0,26 \mathrm{abc}$

CV (\%)

17,8

18,7

19,3

${ }^{1}$ Médias seguidas pela mesma letra, na coluna não diferem significativamente, ao nível de $5 \%$ segundo o teste de Duncan.

categorias são pragas que influenciam o estabelecimento da cultura, isto é, podem reduzir o número ideal de plantas por unidade de área. Portanto, nesses casos os inseticidas devem ser aplicados antes, durante ou imediatamente após o plantio, utilizando o tratamento de semente, a aplicação no sulco de plantio ou a pulverização em pré-emergência, dependendo da espécie de praga presente. Entretanto o tratamento da semente por requerer uma menor quantidade de ingrediente ativo do que a aplicação no sulco via pulverização ou aplicação de produtos granulados, é considerado um processo mais econômico (Radford \& Allsopp 1987). No geral, a utilização de um ou outro produto misturado à semente, à exceção do carbaryl, propiciou substancialmente um maior estabelecimento de plantas por unidade de área. Resultados semelhantes foram obtidos por Robertson (1991), que observou um aumento significativo nas taxas de estabelecimento de plântulas em algodão e sorgo pelo tratamento da semente embora não houvesse alta mortalidade larval da praga avaliada. Segundo esse autor e Drinkwater et al. (1990) alguns produtos químicos como o carbofuran e furathiocarb avaliados em seus experimentos, apesar de darem baixa mortalidade para alguns insetos de solo, propiciam uma alta taxa de proteção à plântula em função da ação significativa de repelência. Dessa maneira a utilização do critério de plantas estabelecidas em função do tratamento químico como aqui apresentado foi adequado, principalmente porque representa a ação protetora dos produtos sobre o complexo de pragas subterrâneas. Critério semelhante foi utilizado por Cruz et al. (1983) que encontraram um aumento de $10 \%$ no número de plantas emergidas de parcelas cujas sementes de milho foram tratadas com inseticidas.

Inseticidas têm sido avaliados para o controle da lagarta-elasmo em diversas culturas incluindo além do milho (All et al. 1979, Cruz et al. 1983), sorgo granífero (Gardner \& All 1982), soja (Reynolds et al. 1959), amendoin (Arthur \& Arant 1956, French \& Morgan 1972) e ervilha (Chalfant 1976). Todos esses autores revelaram que $o$ 
inseto é de difícil controle com inseticida, especialmente através de pulverizações exigindo a utilização de altas doses dos produtos (All \& Gallaher 1977). O controle é também dificultado em solo seco (Reed et al. 1987). A eficiência do tratamento de sementes já tinha sido apontada como a melhor alternativa de controle da praga na cultura do milho (Cruz et al. 1983). Ainda hoje, esse tratamento tem sido eficiente, como mostrado no presente trabalho, pois a quantidade de plantas mortas pelo inseto nas parcelas sem tratamento da semente foi o triplo daquela obtida nas parcelas tratadas com os inseticidas sistêmicos.

Poucos trabalhos têm sido realizados com a lagarta-do-cartucho, $S$. frugiperda em relação ao controle químico via tratamento de sementes na cultura do milho. Bowling (1968) na cultura da soja, estudando várias doses de inseticidas via tratamento de sementes, salientou que o carbofuran na dose de $126,249,375$ e 501 gramas do princípio ativo para $100 \mathrm{~kg}$ de sementes foi efetivo no controle de lagartas recém-nascidas, colocadas sobre a planta três semanas após o plantio. Em milho aparentemente essa eficiência não foi verificada mesmo utilizando uma dose de $700 \mathrm{~g}$ do princípio ativo para $100 \mathrm{~kg}$ de sementes.

No geral, a utilização de um ou outro produto misturado a semente, à exceção do carbaryl, propiciou substancialmente um maior estabelecimento de plantas por unidade de área, principalmente pela atuação sobre aquelas pragas que causam mortalidade à planta, isto é sobre pragas subterrâneas e sobre a lagartaelasmo. Em função do maior número de plantas estabelecido e em menor escala, pela atuação de alguns produtos sobre a infestação da lagartado-cartucho, houve diferença significativa na produção. $\mathrm{O}$ rendimento médio obtido de parcelas tratadas foi de $4.990 \mathrm{~kg} / \mathrm{ha}$, ou seja, $1.080 \mathrm{~kg} / \mathrm{ha}$ a mais $(27,6 \%)$ do que o rendimento obtido de parcelas testemunhas $(3.910 \mathrm{~kg} / \mathrm{ha}$ ) mostrando que o tratamento químico da semente de milho com inseticidas para o controle de pragas iniciais é uma tecnologia viável. Apesar de ainda ser necessário um maior volume de informações, os dados obtidos indicaram uma tendência de se ter melhores resultados no estabelecimento da cultura do milho quando os inseticidas estavam misturados com micronutrientes, especialmente para o princípio ativo thiodicarb.

\section{Literatura Citada}

All, J.N. \& R. N. Gallaher. 1977. Detrimental effect of non-tillage corn cropping systems involving insecticides, hybrids, and irrigation on lesser cornstalk borer infestations. J. Econ. Entomol. 70: 361-365.

All, J. N., R.N. Gallaher \& M. D. Jelium. 1979. Influence of planting date, preplanting weed control, irrigation, and conservation tillage practices on efficacy of planting time insecticide applications for control of lesser cornstalk borer in field corn. J. Econ. Entomol. 72: 265-268.

Arthur, B.W. \& F.S. Arant. 1956. Control of soil insects attacking peanuts. J. Econ. Entomol. 49: 68-71.

Bowling, C.G. 1968. Systemic insecticide seed treatment tests on soybeans. J. Econ. Entomol. 61: 1224-1227.

Carvalho, R.P.L. 1970. Danos, flutuação da população, controle e comportamento de Spodoptera frugiperda (J.E. Smith, 1797) e suscetibilidade de diferentes genótipos de milho, em condições de campo. Tese de doutorado, ESALQ/USP, Piracicaba, $170 \mathrm{p}$.

Chalfant, R.B. 1976. Chemical control of insect pests of the southern pea in Georgia. Univ. Ga. Res. Bull. 179. 31 pp.

Cruz, I., L. J. Oliveira \& J.P. Santos. 1983. Efeito de diversos inseticidas no controle da lagarta-elasmo em milho. Pesq. Agropec. Bras. 18: 1293-1301. 
Cruz, I \& F. T. Turpin, 1982. Efeito da Spodoptera frugiperda em diferentes estadios do crescimento da cultura do milho. Pesq. Agropec. Bras. 17: 355-359.

Cruz, I \& F. T. Turpin, 1983. Yield impact of larval infestation of the fall armyworm Spodoptera frugiperda (J.E. Smith) to mid-whorl growth stage of corn. J. Econ. Entomol. 76:1052-1054.

Drinkwater, T.W., J. H. Giliomee \& K. L. Prinngle. 1990. Efficacy of soil-applied and seed dressing insecticides for the control of false wireworm, Somaticus species (Coleoptera: Tenebrionidae) attacking maize. Crop Prot. 9:128-130.

French, J.C. \& L.W. Morgan. 1972. Damage and control of the lesser cornstalk borer, Elasmopalpus lignosellus (Zeller) on peanuts. J. Am. Peanut Res. Educ. Assoc. 4: 41-42.

Gardner, W.A. \& J.N. All. 1982. Chemical control of the lesser cornstalk borer in grain sorghum. J. Ga. Entomol. Soc. 17: 167-71.

Petty, H.B. 1967. How to control soybean insects. Farm Tech. 23: 43-47.

Radford, B.J., P. G. Allsopp. 1987. Use of insecticides and a press wheel to control soil insects affecting sorghum and sunflower establishment in southern Queensland. J. Aust. Entomol. Soc. 26: 161-167.

Reed, T.D., J. W. Todd \& M. H. Bass. 1987. A new technique for determining the effects of soil moisture on insecticide efficacy against lesser cornstalk borer larvae. J. Entomol. Sci. 22: 169-174.

Reynolds, H.T., L.D. Anderson \& L.A. Andres. 1959. Cultural and chemical control of the lesser cornstalk borer in Southern California. J. Econ. Entomol. 52: 63-66.

Robertson, L.N. 1991. Soil-insecticide bioassays and seed treatments for soilinsect control in central Queensland, Australia. Crop Prot. 10: 293-298.

Waquil, J.M., I. Cruz, P.A.Viana, J. P. Santos, F. H. Valicente \& W. J. R. Matrangolo. 1992. Levantamento de pragas subterrâneas e sua importância na redução da população de plantas. p. 133144. In: Anais Reunião Sobre Pragas Subterrâneas dos Países do Cone Sul, 2. CNPMS, Sete Lagoas, MG, 194p.

Recebido em 07/04/95. Aceito em 28/03/96. 